\title{
Corrigendum and Addendum to my Paper "On the Real Part of an Entire Function, Its Derivative and Its Lower Order"
}

\author{
By
}

T. V. Lakshminarasimhan, Madras, India

(Received May 10, 1968)

It was brought to our notice by Dr. S. JAENISCH that Lemma 2 and the condition that $\operatorname{Re}\left[z f^{\prime}(z)\right]>0$ for entire functions $f(z)$ with $f^{\prime}(z)$ denoting the derivative of $f(z)$ do not hold good thereby vitiating our argument in a part of the proof of our Theorem $B$ in [1]. This theorem can however be reformulated as given below so as to obtain a counterpart of Rajagopal's theorem ([2], Theorem I).

Theorem. If $f(z)$ is an entire function of lower order $\lambda(0 \leq \lambda \leq \infty)$ then

$$
\begin{gathered}
\liminf _{r \rightarrow \infty} \log \left[A_{1}^{*}(r) / A^{*}(r)\right] / \log r \leq \lambda \\
\liminf _{r \rightarrow \infty} \frac{\log \log A_{1}^{*}(r)}{\log r} \geq \lambda
\end{gathered}
$$

where $A_{1}^{*}(r)=\underset{|z|=r}{\operatorname{Max} . \mid}\left|\operatorname{Re}\left[z f^{\prime}(z)\right]\right|$ and $A^{*}(r)=\underset{|z|=r}{\operatorname{Max}}|\operatorname{Re} f(z)|$.

The proof depends on deriving the two inequalities

$$
\text { (a) } \liminf _{r \rightarrow \infty} \frac{\log \lambda(r)}{\log r} \leq \lambda \text {; (b) } \liminf _{r \rightarrow \infty} \frac{\log \log A_{\mathbf{1}}^{*}(r)}{\log r} \geq \lambda
$$

with $\lambda(r)=\left[A_{1}^{*}(r) / A^{*}(r)\right]$. The inequality (a) is easily derived as on page 247 in [1] above where we have to replace Max. Re $z f^{\prime}(z)$ by $A_{\mathbf{1}}{ }^{*}(r)$ with the steps preceding the inequality (5) being replaced by

$$
A_{1}^{*}(r) \leq 2 \nu\left(e_{n}\right) A^{*}\left(e_{n}\right) \leq 2 e_{n}^{\lambda+\varepsilon} A^{*}\left(e_{n}\right)
$$

where $\varepsilon$ is arbitrary and the sequence $\left\{e_{n}\right\}$ is derived from the set $E \cap F$ where $F$ denotes the set of points $r$ which lie outside a set of exceptional segments in which for $r>R$, the variation of $\log r$ is less 
than $K v(R / k)^{-1 / 12}$ and $E$ is the set defined as in [1], page 247. Now to derive the inequality $(b)$ we need the following lemmas.

Lemma 1. In the preceding notation $A^{*}(r)$ is an increasing convex function of $\log r$ in the interval $r_{1} \leq r \leq r_{2}$.

Proof. This follows easily since $u(z)=|\operatorname{Re} f(z)|$ is subharmonic in $D: r_{1} \leq|z|=r \leq r_{2}$ and it is well known that max $u(z)$, for any subharmonic function $u \equiv u(z)$, is a convex function of $\log r$.

Lemma 2. For $r>r_{0}$

$$
\operatorname{Max}_{|z|=r} \operatorname{Re} z f^{\prime}(z) \mid \geq A^{*}(r) /(\log r) .
$$

Proof. With $A^{*}(r)=\left|\operatorname{Re} f\left(r e^{i \theta}\right)\right|$,

$$
\begin{aligned}
\left|\operatorname{Re} z f^{\prime}(z)\right| & =\left|\operatorname{Re}\left[r e^{i \theta} \lim _{\varepsilon \rightarrow 0} \frac{f\left(r e^{i \theta}\right)-f\left(r(1-\varepsilon) e^{i \theta}\right)}{\varepsilon r e^{i \theta}}\right]\right| \\
& =\left|\lim _{\varepsilon \rightarrow 0} \frac{\operatorname{Ref}\left(r e^{i \theta}\right)-\operatorname{Ref}\left(r(\mid-\varepsilon) e^{i \theta}\right)}{\varepsilon}\right| \\
& \geq \lim _{\varepsilon \rightarrow 0} \frac{A^{*}(r)-A^{*}(r-r \varepsilon)}{\varepsilon} \\
& =\lim _{\varepsilon \rightarrow 0} \frac{g(r) \log r-g(r-r \varepsilon) \log (r-r \varepsilon)}{\varepsilon} \\
& \geq g(r) \lim _{\varepsilon \rightarrow 0} \frac{\log r-\log (r-r \varepsilon)}{\varepsilon} \\
& =g(r) \lim _{\varepsilon \rightarrow 0} \frac{-\log (1-\varepsilon)}{\varepsilon} \\
& =g(r)=A^{*}(r) / \log r .
\end{aligned}
$$

In the above steps $g(r)=A^{*}(r) / \log r$, an increasing function of $\log r$ by Lemma 1 .

Lemma 3. For the entire function $f(z)$ of order $\varrho$ and lower order $\lambda$ we have

Since

$$
\lim _{r \rightarrow \infty} \sup \frac{\log \log A^{*}(r)}{\log r}=\frac{\varrho}{\lambda} \quad(0 \leq \lambda, \varrho \leq \infty) .
$$

$$
\left|a_{n}\right| r^{n} \leq 2 A^{*}(r) \leq \underset{|z|=r}{2 \operatorname{Max}}|f(z)|=2 M(r), n>0,
$$


we have, if $\mu(r)$ is the maximum term in the power series for $f(z)$ corresponding to $|z|=r$,

$$
\mu(r) \leq 2 A^{*}(r) \leq 2 M(r)
$$

from which we derive

$$
\limsup _{r \rightarrow \infty} \frac{\log \log A^{*}(r)}{\log r}=\varrho
$$

and similarly for the lower order $\lambda$.

Proof of Theorem. Lemmas (2) and (3) yield

$$
\liminf _{r \rightarrow \infty} \frac{\log \log A_{1}^{*}(r)}{\log r} \geq \lambda
$$

which is inequality $(b)$. Since inequality $(a)$ is already established the theorem is completely proved.

Our thanks are due to $D_{\text {r. }}$ S. JAENISCH for his comments.

\section{References}

(l) Lakshmmarasimenan, T. V.: On the real part of an entire function, its derivative and its lower order. Mh. Math. 70, 244-247 (1966).

(2) RAJAGOPAL, C. T.: On an asymptotic relation between an entire function, its derivative and their order. Mh. Math. 66, 339-345 (1962).

Department of Mathematics Madras Christian College Madras-59, India. 